\title{
I viaggi in un romanzo e i viaggi di un romanzo nel basso medioevo. II caso del Joufroi de Poitiers
}

Roberta Manetti

Anche il filologo che non si sia mai occupato direttamente di letteratura odeporica legge molti racconti di viaggio, nelle opere letterarie medievali. Difficilmente i personaggi dei romanzi, specie quelli maschili, sono sedentari e appaiono anzi non di rado impegnati in guerre, crociate, passaggi da una corte all'altra, queste del Graal o ricerche dell'amata o dell'amato rapiti o venduti o in fuga da qualche persecutore, oppure in avventurosi viaggi per conquiste territoriali o per commerci; nei romanzi in versi del XII e del XIII secolo ci si può muovere in cerca d'avventure o per compiere una missione o per andare a completare la propria educazione cavalleresca in qualche corte regale prestigiosa: la casistica è ampia, come lo sono gli spazi percorsi, che talvolta si ispirano a reali itinerari compiuti dall'autore o si rifanno a racconti di viaggio preesistenti, magari opera di autori in qualche modo evocati nella finzione narrativa.

È quel che accade, ad esempio, nel Joufroi de Poitiers, un bizzarro romanzo in antico francese, mutilo in fine, il cui protagonista nei 4613 octosyllabes a rima baciata superstiti viaggia moltissimo. La figura di Joufroi richiama quella del primo trovatore, Guglielmo VII conte di Poitiers (oltre che duca d'Aquitania), come viene tratteggiata dai cronisti e dalla vida duecentesca, che pare rifarsi, genealogia a parte, ad alcuni componimenti dell'arguto conte-duca, e specialmente a Farai un vers pos mi sonelh (BdT,183.12), che racconta in prima persona un viaggio in incognito per sedurre con l'inganno le mogli di due vassalli; l'anoni-

Roberta Manetti, University of Florence, Italy, roberta.manetti@unifi.it

FUP Best Practice in Scholarly Publishing (DOI 10.36253/fup_best_practice)

Roberta Manetti, I viaggi in un romanzo e i viaggi di un romanzo nel basso medioevo. II caso del Joufroi De Poitiers, pp. 157-164, (c) 2021 Author(s), CC BY 4.0 International, DOI 10.36253/978-88-5518-4670.14, in Michela Graziani, Lapo Casetti, Salomé Vuelta García (edited by), Nel segno di Magellano tra terra e cielo. II viaggio nelle arti umanistiche e scientifiche di lingua portoghese e di altre culture europee in un'ottica interculturale, (c) 2021 Author(s), content CC BY 4.0 International, metadata CC0 1.0 Universal, published by Firenze University Press (www.fupress.com), ISSN 2704-5919 (online), ISBN 978-88-5518467-0 (PDF), DOI 10.36253/978-88-5518-467-0 
mo autore della vida sostanzialmente riassume il vers ${ }^{1}$, tratteggiando il ritratto di un valente cavaliere e insaziabile dongiovanni, con spiccatissima inclinazione alla burla, come il personaggio eponimo del Joufroi de Poitiers. Questi percorre in lungo e in largo i territori insulari e continentali che nella seconda metà del XII secolo furono dei Plantageneti, con una puntata nel Ducato di Borgogna.

Il tema del viaggio è così centrale che perfino l'anonimo autore, che intreccia in più di un punto alla storia narrata una vicenda amorosa pseudo-autobiografica e fa sentire molto la sua presenza dall'inizio alla fine, finge che la materia gli venga da un soggiorno lontano da casa, nella zona di Montpellier: qui, nella cattedrale di San Pietro e Paolo a Magalona, avrebbe rinvenuto un manoscritto in latino con una storia che avrebbe prima tradotto e poi verseggiato. Un dettaglio indubbiamente topico, ma come vedremo, non è certo scelto a caso il nome del luogo, specie se a questo viaggio (forse niente affatto immaginario) dell'autore si accostano altri itinerari, reali o fittizi, di personaggi, di testi e di manoscritti.

Perché la storia di Joufroi, conte di Poitiers, non è stata trovata nel Poitou o in una qualunque parte dell'antico dominio dei duchi d'Aquitania, ma a Montpellier, che al tempo del primo trovatore non era nemmeno ancora una città importante ${ }^{2}$ ? Forse nella scelta c'è qualcosa di allusivo, visto che a metà del Duecento, mentre Poitiers era di fatto francese ${ }^{3}$, Montpellier era saldamente in mano aragonese. Dopo il matrimonio, nel 1204, dell'erede Maria col re Pietro II d'Aragona, fu destinata al primogenito maschio della coppia, che nacque solo nel 1208; in caso di morte dell'erede e sua, Maria aveva designato per la signoria della città i cugini Arnaut e Raimon II de Rocafolh, già indicati come successori nel testamento del padre di Maria, Guglielmo VIII, in caso di estinzione della discendenza diretta. Fra i vari titoli nobiliari di Arnaut c'era quello di signore di Algues: è lui il sener d'Alga che l'anonimo autore del bellissimo romanzo occitano, noto sotto il titolo moderno di Flamenca, nomina (al v. 1724) e con cui pare avere una certa familiarità ${ }^{4}$ I Rocafolh non divennero signori di Montpellier, perché Giacomo I sopravvisse all'infanzia, il che all'epoca non era proprio scontato, e, se Pietro II aveva quasi abbandonato la città ai consoli dopo il 1207, il figlio vi ristabilì progressivamente i suoi diritti, insediandovi già dal 1218 dei suoi fedelissimi col titolo di luogotenenti, cioè governatori che

1 «Lo coms de Peiteus si fo uns dels majors cortes del mon e dels majors trichadors de domnas, e bons cavaliers d'armas e larcs de domnejar; e saup ben trobar e cantar. Et anet lonc temps per lo mon per enganar las domnas» (Boutière, Schutz 1973, 7, che traducono: «Le comte de Poitiers fut un des [hommes] les plus courtois du monde et l'un des plus grands trompeurs de femmes; il fut bon chevalier d'armes et généreux en galanterie; il sut bien "trouver" et chanter; et il alla longtemps à travers le monde pour tromper les dames»).

2 In seguito fu probabile centro di irradiazione di materiale lirico guglielmino (cfr. Meneghetti 2014).

3 Filippo Augusto aveva conquistato la città quattro mesi dopo la morte di Eleonora d'Aquitania, nel 1204; dal 1241 al 1271 il titolo di conte di Poitiers fu rivestito da Alfonso, fratello minore del re di Francia.

4 Cfr. Manetti 2008, 35-7 e 2018a, 45. 
lo rappresentassero in sua assenza e sorvegliassero i balivi locali, strettamente legati ai consoli ${ }^{5}$. Il luogotenente abitava l'antico palazzo signorile dei Guillem de Montpellier, che Giacomo fece restaurare soggiornando spesso nella città materna, specie dal 1231; durante una permanenza dal dicembre 1236 al giugno 1237 prestò omaggio al vescovo di Magalona (la stessa evocata nel Joufroi de Poitiers), testimone Arnaut de Rocafolh. Dall'inizio degli anni Cinquanta ${ }^{6}$ la carica di luogotenente fu ricoperta per parecchi anni da Guillem de Rocafolh, figlio illegittimo prematrimoniale di Arnaut, poi legittimato e nobilitato dal re d'Aragona, di cui era il braccio destro in guerra e in pace almeno fin dal tempo della presa di Valencia, nel 1238. A lui, oltre che allo stesso re ${ }^{7}$, occhieggia probabilmente il protagonista maschile positivo di Flamenca, il giovane valoroso e astutissimo Guillem, che, travestito da chierico, riesce a divenire l'amante della protagonista, benché il marito Archimbaut de Borbon, reso folle di gelosia dalle insinuazioni della regina di Francia durante la festa di nozze, l'abbia reclusa in una torre (ma la fama della sua bellezza e delle sue virtù arriva a Guillem, che si innamora da lontano). La citazione del sener d'Alga è un indizio forte per dedurre l'ambiente di composizione del romanzo occitano: giustappunto Montpellier, che nel XIII secolo era una città culturalmente molto vivace e ben lontana dall'annessione alla Corona di Francia, avvenuta solo a metà del Trecento. Era, di conseguenza, una possibile zona franca per la produzione e la prima circolazione di opere pervase di satira anticapetingia anche feroce ${ }^{8}$ : tale è Flamenca, che mette impietosamente alla berlina un personaggio del calibro di Arcibaldo di Borbone senza nemmeno velarne il nome e il casato, come di norma si faceva coi grandi personaggi viventi dileggiati in letteratura; l'autore lo fa diventare animalesco e ridicolo fin dall'inizio, lo fa tradire dalla moglie nonostante le precauzioni e lo dipinge alla fine come rinsavito, ma sempre cornuto (cogotz), ingannato dall'amante della consorte che gli si finge amico e alleato e, prima ancora, da un giuramento ambiguo di lei, Flamenca, nome che significa la sfolgorante e insieme la fiamminga, un personaggio senza esatta corrispondenza nella realtà e che simboleggia il Meridione, benché sia figlia di un conte che parrebbe stare nel Nord-Est ${ }^{9}$.

5 È il balivo che esercita il potere in nome del re-signore nella città di Montpellier, ma quest' $\mathrm{i}$ stituzione è strettamente connessa a quella del consolato e dunque sfugge, più o meno, al controllo del signore: per questo Giacomo I istituì i luogotenenti, con ruolo soprattutto di rappresentanza, facendo da raccordo fra il re e i suoi vassalli, ma anche di sorveglianza. Dal 1252 al 1259 e dal 1263 al 1267; dettagli e riferimenti bibliografici in Manetti 2018a.

7 Cui almeno fino al 1242 (e qualcuno anche oltre) i meridionali, progressivamente spossessati o sempre più minacciati dai francesi nel corso della crociata antialbigese, guardavano come a una sorta di Messia liberatore.

8 Questo aspetto viene sviluppato in Manetti 2018a.

9 Non è tuttavia il conte di Fiandra, del quale la famiglia di Flamenca frequenta la corte e che compare nell'ultima giostra superstite del torneo finale del romanzo, interrotta dalla perdita delle ultimissime carte dell'unico manoscritto. 
Flamenca è un personaggio che rappresenta, fra le altre cose, anche il filo che parrebbe piuttosto diretto fra il Sud-Ovest, specie quello non caduto in mano francese, e il Nord-Est, zona di "fronda" anticapetingia: una fronda che serpeggiava un po' in tutta la parte orientale della Francia, compresa quella di confine fra area d'oïl e area franco-provenzale della quale parrebbe originario l'anonimo autore del Joufroi de Poitiers; questi non solo, con lo scegliere un eroe aquitano e con l'affermare di aver trovato di persona la storia a Montpellier, evidenzia il filo in questione, ma ricalca le modalità d'esercizio della satira dell'anonimo occitano prendendo di mira un esponente dell'alta nobiltà più vicina alla famiglia reale, Guy de Châtillon e Saint-Pol, che, seppur non nominato, sarà stato riconosciuto dal pubblico coevo alla stesura del romanzo nel marito gelosissimo di una bella dama di cui Joufroi ${ }^{10}$ si è invaghito da lontano, sentendone lodare la bellezza ${ }^{11}$; travestito da chierico, inganna il marito, che credendolo un santo eremita incoraggia la frequentazione della moglie per tutti gli altri reclusa in una torre, e seduce la dama. Il nome di lei è Agnes de Tornuerre, ovvero Tonnerre, famiglia strettamente imparentata con quella di Borbon: una figlia di Agnés de Tonnerre e di Guy de Châtillon e Saint-Pol, Yolande, aveva sposato Archimbaut IX, il figlio dell'Archimbaut dileggiato in Flamenca: i due cornuti dei due romanzi sono consuoceri.

Cornuto è un insulto molto più forte di quanto possa parere a un cittadino evoluto del XXI secolo, ed è polisemico: l'appellativo si rivolge non solo ai tradi$\mathrm{ti}$ in ambito domestico, ma anche ai traditori in ambito esterno; tale, agli occhi di un meridionale, può apparire Archimbaut, signore di una regione occitana, l'Alvernia, ma che da sempre sta al fianco dei capetingi, sia durante la cosiddetta crociata del re, quando Luigi VIII prese Avignone e morì di dissenteria poco dopo, l'8 novembre 1226, con Archimbaut al capezzale, sia durante gli ultimi moti del 1241-1242, che si chiusero con le battaglie di Taillebourg e Saintes, nelle quali Archimbaut era a capo dell'esercito di Luigi IX, facendo peraltro subito dopo, nel pieno dell'estate del 1242 , la stessa fine ${ }^{12}$ che aveva fatto Luigi VIII. La mia ipotesi è che Flamenca sia stato finito poco dopo, dato che il torneo su cui il manoscritto mutilo si interrompe mima con modi parodici alcuni avvenimenti salienti degli anni 1241-1242.

Alla corte del re d'Aragona, che sarà giustappunto quella di Montpellier, e in un'epoca non distante fu scritto, come dichiara esplicitamente l'autore, anche l'altro grande romanzo occitano superstite, Jaufre, il cui protagonista porta la variante occitana del nome Joufroi, eroe di un romanzo che, come si è accennato, si finge ispirato dal ritrovamento di un manoscritto a Montpellier e riprende da un

10 Che, dopo il ritorno dal primo viaggio in Inghilterra, a Poitiers si ferma molto poco e vaga in cerca di tornei tra Île-de-France, Gâtinais, Champagne, Bretagna, prima di aver lo spunto per l'avventura a Tonnerre.

11 Come Guillem in Flamenca, ma anche come il Jaufre Rudel consegnato a un durevole mito dalla vida duecentesca edita in Boutière, Schutz 1973, 16.

12 Morì di dissenteria in una delle ricorrenti epidemie che scoppiavano fra gli eserciti, senza distinguere tra vincitori e vinti. 
romanzo scritto davvero probabilmente a Montpellier, Flamenca, il motivo della dama rinchiusa nella torre dal marito geloso e del giovane astutissimo amante che riesce ad arrivarci in incognito, travestito da chierico, oltre che la satira ai danni di un potente signore filocapetingio. Tornuerre è una leggera storpiatura, probabilmente d'autore, per Tonnerre (nell'attuale regione della Borgogna-Franca Contea, il punto continentale più a est in cui si spinge Joufroi); per un lettore o un ascoltatore della metà del XIII secolo o poco dopo la leggera deformazione (anche nella variante Torneure) non maschera nulla e il nome della dama avrà evocato immediatamente la contessa Agnès II di Tonnerre, Auxerre e Nevers ${ }^{13}$ (1205-1225), moglie ${ }^{14}$ del nipote di Luigi VI di Francia, Gui III de Châtillon, II come conte di Saint-Pol, ucciso durante l'assedio di Avignone nel 1226. Fra i migliori amici di Luigi VIII, aveva combattuto al suo fianco anche nel 1223, quando il re, appena consacrato, aveva preso con le armi quasi tutti i possedimenti inglesi in Aquitania, fra Poitou, Saintonge, Périgord, Angoumois e parte del Bordolese; ai Plantageneti rimase la Guienna, ovvero la regione invocata nel grido di guerra dell'armata del conte di Poitiers contro il conte di Tolosa nel romanzo.

Anche nel Joufroi de Poitiers si ridicolizza dunque un esponente dell'alta nobiltà più fedele ai Capetingi, addirittura morto per loro, come Archimbaut. Ed ecco che il viaggio a Montpellier dell'autore del romanzo francese potrebbe semplicemente simboleggiare il viaggio verso est del romanzo occitano, di cui si condivide lo spirito anticapetingio e si replica con qualche variazione la modalità di esercitare una satira delle più feroci, dando di cornuto, con tutte le implicazioni che comporta, a un nobiluomo intimo del re e con ruoli militari di grande rilievo nella crociata antialbigese. Nel Joufroi de Poitiers il re di Francia fa una rapida apparizione in una scena di torneo, dove il conte di Poitiers lo prende di mira, lo abbatte con un sol colpo e gli porta via il cavallo: anche questo è un modo per esprimere scarsa stima e anzi spiccata antipatia per i Capetingi ${ }^{15}$.

Con l'Inghilterra l'autore sembra avere una certa confidenza; la geografia del romanzo è fatta di luoghi reali e riconoscibili ${ }^{16}$, quasi tutti compresi nei territori

13 Nevers è anche il casato che evoca, con alterazione minima, il nome dell'eroe di Flamenca, Guillem de Nivers.

14 Inizialmente fidanzata col primogenito, morto bambino, del futuro Luigi VIII e di Bianca di Castiglia (con questa unione il nonno dello sposo, Filippo Augusto, sperava di portare Nevers, Auxerre e Tonnerre direttamente sotto la Corona).

15 Incluso forse il conte di Poitiers in carica al momento della stesura dei due romanzi, il fratello prediletto di Luigi IX, Alfonso, alla cui investitura nel 1241 il conte della Marca Ugo di Lusignano scatenò la ribellione, coinvolgendo il re d'Inghilterra, ma piantandolo in asso al momento delle due battaglie risolutive, in Aquitania.

16 A parte l'immaginario Paese di Cuccagna dietro cui Joufroi maschera il suo vero casato durante la prima delle sue spedizioni in incognito (da vero trickster, si traveste per ben tre volte: da sire di Cocagne, da santo eremita, da cavaliere di rango modesto e per metà borghese); un mitico paese dell'abbondanza che con vari nomi compare anche nell'antichità, ma che riceve questa denominazione proprio nella letteratura francese del XIII secolo (cfr. Väänänen 1947), che indica questo testo, datato alla metà del secolo, come il primo in cui compare. Non siamo cronologicamente lontani dal Joufroi de Poitiers. 
dei Plantageneti, insulari e continentali. Joufroi va in Inghilterra due volte, sempre partendo da Dieppe, affacciata sulla Manica e inclusa nel ducato di Normandia; una città fedele ai re normanni d'Inghilterra, tanto che nel 1195 i francesi la assediarono e la presero, incendiandone le navi. Al primo viaggio Joufroi sbarca a Southampton (Sozantone, 147) e da lì prosegue per York, dove il re tiene corte, poi si offre come campione per la regina diffamata dal siniscalco e il re fissa il duello a Guincestre, ovvero Winchester. Da lì, il giorno seguente a quello della vittoria, Joufroi deve rimpatriare, avendo ricevuto la notizia della morte del padre. Rientrato a Poitiers e compiuta l'impresa erotica a Tonnerre, il conte si trova a scommettere col suo fido vassallo Robert su chi sia più valoroso: per stabilirlo, partono in incognito per una sfida ad armi pari in un posto dove nessuno li identifichi e scelgono l'Inghilterra, visto che ormai Joufroi, ragazzino all'epoca del primo viaggio, è adulto e irriconoscibile. La seconda traversata riassume in due versi il viaggio da Dieppe a Lincoln, dove il re è in guerra col re di Scozia.

Joufroi per mascherarsi in quest'avventura inglese sceglie un curioso pseudonimo, Girart / Giraut de Berri: lo portava un chierico di origini nobili, elemosiniere di Enrico II dal 1184, noto anche come Giraldus Cambrensis, che viaggiò molto al seguito di principi e altissimi prelati e descrisse le terre visitate e le imprese dei personaggi che accompagnava in opere poi molto lette, come l'Itinerarium Cambriae, la Descriptio Cambriae o la Topographia Hibernica; è sempre lui che, in un De principis instructione, racconta di come Guglielmo conte di Poitiers rapì al suo vassallo la moglie, «quæ Mauberium dicta fuit», per farne la sua consorte di fatto (Bartlett 2018, cap. XXVII).

Uno strano modo di mimetizzarsi, quello di assumere il nome di un personaggio così eminente della corte d'Inghilterra il cui ricordo, anche grazie alle sue numerose opere letterarie, travalicò di parecchio gli anni in cui visse. Il Joufroi de Poitiers sovrappone personaggi di più secoli, mischiando un po' le carte, lasciando tuttavia sempre capire che non si parla solo del tempo andato: il re d'Inghilterra si chiama Enrico e adombra Enrico II Plantageneto, ma dal 1216 al 1272 è stato re un altro Enrico Plantageneto, quello sconfitto a Taillebourg e Saintes nel 1242; il conte padre di Joufrois si chiama Richiers, come Riccardo Cuor di Leone, conte di Poitiers dal 1189, figlio di Enrico II e di Eleonora d'Aquitania, che ebbero tra i figli anche un Goffredo; Alienors è il nome della madre di Joufrois, mentre la regina d'Inghilterra si chiama Halis, come la figlia di secondo letto di Luigi VII di Francia, che Enrico II fece venire in Inghilterra come fidanzata per il figlio Riccardo e che pare sia divenuta invece la sua amante, ma anche come la prima moglie di Archimbaut VIII, Alix de Forez: così, en passant, visto che Joufroi de Poitiers sedurrà anche la regina d'Inghilterra, gli si dà del cornuto anche in questo romanzo.

La toponomastica inglese che compare nel Joufroi de Poitiers non era certo sconosciuta al pubblico francese del XIII secolo: sono città che compaiono nei romanzi precedenti, da Chrétien de Troyes in poi, e le informazioni potevano venire all'autore per via letteraria; ma è possibile che egli sia stato, oltre che sicuramente a Tonnerre, anche Oltremanica e che l'esperienza della corte inglese sia diretta. Il nascondersi di Joufroi dietro un nome che tutti, in quella corte, 
avrebbero riconosciuto, può essere un altro dettaglio ironico: dalla satira non si salva nessuno, nemmeno il re d'Inghilterra, poco acuto, incapace di cavarsela da solo in battaglia - mai avrebbe vinto i nemici, senza l'aiuto di Joufroi - come lo fu il re Enrico III a Taillebourg e Saintes, tradito dal figliastro Ugo di Lusignano che non scese in campo e corse a far mea culpa dal sovrano francese (anche il re d'Inghilterra del romanzo finisce tradito, sia pure dalla moglie).

Il messaggio dell'autore del Joufroi, che scrive certo dopo i fatti del 1242, dato che la riscossa in armi della Provenza gli pare un adynaton comparabile al disprezzo della fede da parte di Dio, alla redenzione di Giuda e al maggior prezzo dell'argento rispetto all'oro ${ }^{17}$, non appare tanto dissimile da quello veicolato dall'autore di Flamenca, romanzo che verosimilmente conosce e assimila: le armi migliori per ottenere o recuperare qualcosa, vista l'impossibilità di vincere manu militari, sono l'astuzia e la dissimulazione.

Oggi noto solo a pochi specialisti, a suo tempo il Joufroi de Poitiers ha viaggiato, come il Jaufre occitano ${ }^{18}$, dall'area di redazione all'Italia settentrionale: è difatti franco-veneta la patina lasciata da almeno un copista nell'unico manoscritto superstite (già riconosciuta da Meyer 1904): segno che il romanzo ebbe una certa diffusione anche a Sud della Francia ${ }^{19}$.

\section{Riferimenti bibliografici}

Bartlett, R., a cura di. 2018. Gerald of Wales, Instruction for a Ruler / De principis instructione. Oxford: Oxford University Press.

BdT cfr. Pillet, Carstens 1933.

Boutière, J., e A. H. Schutz. 1973. Biographies des troubadours. Paris: Nizet.

17 Così ai vv. 3582-3583: «Riches serez? fait li borgeis; / iche sera quant Deus li reis / non amera foi ne creanche, / et Provence conquerra Franche / par armes sans neguns content, / et or sera plus vil d'argent, / et Judas iert de pechiez quites / quant ce sera que vous me dites» $[\ll$ Sarete ricco?», ribatté il borghese, «Questo accadrà quando Dio che regna non amerà né fede né osservanza, e la Provenza vincerà la Francia con le armi senza nessuna resistenza, e l'oro sarà più vile dell'argento $\mathrm{e}$ Giuda sarà assolto dal peccato, quando accadrà quello che mi dite!» trad. nostra]; reputare la conquista armata (la riscossa?) della Provenza ai danni della Francia possibile quanto lo spregio di foi e creanche da parte di Dio, il ribaltamento della scala di valori tra oro e argento e l'assoluzione di Giuda si colloca forse ancor meglio dopo la morte del conte di Provenza (1245, erede la figlia Beatrice, sposata nel 1246 da Carlo d'Angiò, fratello del re di Francia) e di quello di Tolosa (1249, erede la figlia Giovanna, già sposata ad Alfonso, fratello del re).

18 Uno dei due manoscritti che conservano il Jaufre è stato trascritto difatti nell'Italia settentrionale all'inizio del XIV secolo; italiani anche due dei cinque frammenti noti.

19 È invece del tutto accidentale l'arrivo a Copenhagen, tre secoli or sono, del manoscritto, ora alla Biblioteca Reale, segnato Gl. Kgl. Saml. 3555: forse fu portato via da un conte danese sceso in Veneto per una lunga missione militare (nella zona di Mantova-Verona, a quell'epoca coinvolta nell'assedio di Eugenio di Savoia-Soissons), tanto lunga che si fece raggiungere dalla moglie e generò un figlio a Verona nel 1702; oppure fu comprato in blocco con codici latini e greci proprio da questo figlio, langravio di Dannesjold-Samsøe, che fin dall'adolescenza fu un eruditissimo e appassionato bibliofilo. 
Manetti, R. 2008. Flamenca. Romanzo occitano del XIII secolo. Modena: Mucchi.

Manetti, R. 2018. Joufroi de Poitiers. Romanzo francese del XIII secolo. Alessandria: Edizioni dell'Orso.

Manetti, R. 2018a. "Da Nord-Est a Sud-Ovest e ritorno: Jean Renart, Joufroi de Poitiers e i due grandi romanzi occitani (Jaufre e Flamenca).” Medioevo Europeo 2: 33-72.

Meneghetti, M. L. 2014. "Oltre lo specchio: il Joufroi de Poitiers e la cultura lirica del suo autore.” Summa 4 (Tardor 2014): 62-74.

Meyer, P. 1904. De l'expansion de la langue française en Italie pendant le moyen-âge, in Atti del Congresso Internazionale di Scienze Storiche (Roma, 1-9 aprile 1903), vol. IV, Atti della Sezione III: Storia della letteratura. Roma: Accademia dei Lincei (anche in estratto, accessibile da < gallica.bnf.fr > (10/20).

Pillet, A., e H. Carstens. 1933. Bibliographie der Troubadours. Halle: Niemeyer.

Väänänen, V. 1947, Le fabliau de Cocagne, «Neuphilologische Mitteilungen», 48: 3-36 (ripreso in Väänänen, V. 1981. Recherches et récréations latino-romanes. 375-406. Napoli: Bibliopolis). 